\title{
The Design of Axisymmetric Ducts for Compressible Flow with Vorticity
}

\author{
V. Pavlika
}

\begin{abstract}
In this paper a numerical algorithm is described for solving the boundary value problem associated with axisymmetric, inviscid, compressible and rotational and irrotational flow. The algorithm is capable of calculating the duct wall geometries from prescribed wall velocity distributions. The equations modeling the flow are expressed using the stream function $\psi(x, y)$ and the function $\varphi(x, y)$ as independent variables, where for irrotational flow $\varphi(x, y)$ can be recognized as the velocity potential function, for rotational flow $\varphi(x, y)$ ceases being the velocity potential function but does remain orthogonal to the stream lines, the $x$ and $y$ are the usual axial and radial coordinates in cylindrical coordinates respectively. The technique described is capable of tackling the so-called inverse problem where the velocity wall distributions are prescribed from which the duct geometry is calculated, as well as the direct problem where the velocity distribution on the pressure and suction surfaces are calculated from prescribed geometries. The two different cases outlined in this paper are in fact boundary value problems with Neumann and Dirichlet boundary conditions respectively with results for the Neumann boundary condition only included. The axial velocity and the swirl velocity are prescribed such that vorticity is transported through the duct. The governing second order partial differential is coupled with a set of quasi-linear hyperbolic first order partial differential equations with characteristics parallel to the $\varphi$ and $\psi$ axes, the numerical solution is thus obtained iteratively using finite differences to approximate derivatives.
\end{abstract}

Index Terms-Rotational, compressible flow, Hagen-Poiseuille flow, swirl velocity.

\section{INTRODUCTION}

Designers of annular ducts require numerical techniques for calculating wall geometries from a prescribed velocity distribution. The objective of the prescribed velocity is typically to avoid boundary layer separation see for example [1]. At inlet Hagen-Poiseuille flow is prescribed (along with an appropriate swirl component of velocity) such that the flow is rotational.

This paper describes a numerical algorithm for solving the boundary value problem that arises when the independent variables are $\varphi$ and $\psi$ where $\varphi$ and $\psi$ have been previously defined. The dependent variable $y$, is the radial coordinate and $x$ the axial coordinate. The numerical technique is based on the finite difference scheme on a uniform rectangular mesh. Similar work can be found in [2], [3].

\section{THE DESIGN Plane}

The duct is set in a meridional plane with points in the flow

Manuscript received August 8, 2013; revised January 15, 2014.

V. Pavlika is with University of London, UK (e-mail: vp4@soas.ac.uk). field given in terms of cylindrical coordinates, $(y, \alpha, x))$, with the $\mathrm{x}$ axis the axis of symmetry. The corresponding velocity components are represented by $u_{y}, u_{a}$ and $u_{x}$. The speed $q$ is given by

$$
q=\left(u_{x}^{2}+u_{y}^{2}\right)^{1 / 2}
$$

which may be prescribed on either or both of the duct walls. Previous work in [4] has shown that the governing partial differential equation that the radius y satisfies is given by:

$$
\frac{\partial}{\partial \varphi}\left(\frac{A}{B} \frac{\partial y}{\partial \varphi}\right)+\frac{\partial}{\partial \psi}\left(\frac{B}{A} \frac{\partial y}{\partial \psi}\right)=0
$$

In addition

$$
\frac{\partial}{\partial \varphi}\left(\log _{e}(A)\right)=\frac{\eta}{q^{2}} B
$$

and

$$
\frac{\partial}{\partial \psi}\left(\log _{e}(B)\right)=-\frac{\omega_{\alpha}}{q^{2}} A
$$

where $\psi(x, y)$ is the usual stream function and $w_{a}$ is the component of vorticity perpendicular to the plane, $\varphi(x, y)$ is a function orthogonal to the stream line and calculation of the axial coordinate is given by:

$$
d x=\frac{B}{A} \frac{\partial y}{\partial \psi} d \varphi-\frac{A}{B} \frac{\partial y}{\partial \varphi} d \psi
$$

and the speed is computed from:

$$
\frac{1}{q^{2}}=\frac{1}{A^{2}}\left(\frac{\partial y}{\partial \psi}\right)^{2}+\frac{1}{B^{2}}\left(\frac{\partial y}{\partial \varphi}\right)^{2}
$$

\section{The Numerical Algorithm In the Design Plane}

Rewriting the partial differential equation that $y$ satisfies (equation (1)) as:

$$
\frac{\partial}{\partial \phi}\left(a \frac{\partial y}{\partial \phi}\right)+\frac{\partial}{\partial \psi}\left(b \frac{\partial y}{\partial \psi}\right)=c
$$

with: $a=a(y, \varphi, \psi), b=b(y, \varphi, \psi), c=c(y, \varphi, \psi)$, and where $a, b$ and $c$ are function of the arguments shown. For problems posed in the design plane $c=0$, the $a$ and $b$ will vary 
depending on whether the flow field is compressible, irrotational or swirl free etc. Writing in finite difference form using central differences (with $c \neq 0$ ) gives:

$$
\frac{\partial}{\partial \phi}\left(a \frac{\partial y}{\partial \phi}\right)_{i, j}=\frac{1}{2(\Delta \phi)^{2}}\left[\left(a_{i+1, j}+a_{i, j}\right) y_{i+1, j}-4 a_{i, j} y_{i, j}+\left(a_{i-1, j}+a_{i, j}\right) y_{-i, j}\right]
$$

and

$$
\frac{\partial}{\partial \psi}\left(b \frac{\partial y}{\partial \psi}\right)_{i, j}=\frac{1}{2(\Delta \psi)^{2}}\left[\left(b_{i, j+1}+b_{i, j}\right) y_{i, j+1}-4 b_{i, j} y_{i, j}+\left(b_{i, j-1}+b_{i, j}\right) y_{i, j-1}\right]
$$

Thus at the point $(i \Delta \varphi, j \Delta \psi)$ (to be denoted by $(i, j)$ from now on in this paper), the equation is represented by a computational molecule as:

$$
\begin{gathered}
+N_{i, j} y_{i, j-1} \\
W_{i, j} y_{i-1, j}-C_{i, j} y_{i, j}+E_{i, j} y_{i+1, j}=R_{i, j} \\
+S_{i, j} y_{i, j+1}
\end{gathered}
$$

where the $N, S, E$ and $W$ and $R$ may be identified as

$$
\begin{aligned}
& W_{i, j}=(\Delta \psi)^{2}\left(a_{i, j}+a_{i-1, j}\right) \\
& E_{i, j}=(\Delta \psi)^{2}\left(a_{i+1, j}+a_{i, j}\right) \\
& N_{i, j}=(\Delta \varphi)^{2}\left(b_{i, j-1}+b_{i, j}\right) \\
& S_{i, j}=(\Delta \varphi)^{2}\left(b_{i, j+1}+b_{i, j}\right) \\
& \left.C_{i, j}=4\left((\Delta \varphi)^{2} a_{i, j}+(\Delta \psi)^{2} b_{i, j}\right)\right) \\
& R_{i, j}=2(\Delta \varphi)^{2}(\Delta \psi)^{2} c_{i, j}
\end{aligned}
$$

\section{THE DIFFERENCE EQUATIONS}

Equation (4) applies for $i=1$ to $M ; j=1$ to $N$ on a uniform mesh as described in [5], with special consideration at $j=1$ and $j=N$, so that with Dirichlet boundary conditions, say for $j=N$

$$
\begin{gathered}
+N_{i, N} y_{i, N-1} \\
W_{i, N} y_{i-1, N}-C_{i, N} y_{i, N}+E_{i, N} y_{i+1, N}=R_{i, j}-S_{i, N} y_{i, N+1}
\end{gathered}
$$

with $y_{i, N+1}$ prescribed as the Dirichlet data for $0 \leq i \leq M$. For $j=2$ to $N-1$

$$
\begin{aligned}
& +N_{i, j} y_{i, j-1} \\
W_{i, j} y_{i-1, j} & -C_{i, j} y_{i, j}+E_{i, j} y_{i+1, j}=R_{i, j} \\
& +S_{i, j} y_{i, j+1}
\end{aligned}
$$

and for $j=1$

$$
\begin{aligned}
W_{i, 1} y_{i-1,1} & -C_{i, 1} y_{i, 1}+E_{i, 1} y_{i+1,1}=R_{i, 1}-N_{i, 1} y_{i, 0}= \\
& +S_{i, 1} y_{i, 2}
\end{aligned}
$$

similarly $y_{i, 0}$ prescribed as the Dirichlet data for
$0 \leq i \leq M$

\section{VECTOR FORM OF THE DIFFERENCE EQUATIONS}

The above equations can be written more conveniently in matrix-vector form as:

$$
\begin{aligned}
& {\left[\begin{array}{ccccc}
W_{i, 1} & 0 & 0 & \cdot & \cdot \\
0 & W_{i, 2} & 0 & & \\
& 0 & W_{i, 3} & \cdot & \cdot \\
& & & \cdot & \cdot \\
& & & & W_{i, N}
\end{array}\right]\left[\begin{array}{c}
y_{i-1,1} \\
y_{i-1,2} \\
\cdot \\
\cdot \\
y_{i-1, N}
\end{array}\right] .} \\
& +\left[\begin{array}{ccccc}
-C_{i, 1} & S_{i, 1} & 0 & \cdot & \cdot \\
N_{i, 2} & -C_{i, 2} & S_{i, 2} & 0 & \cdot \\
& N_{i, 3} & -C_{i, 3} & \cdot & \cdot \\
& & & \cdot & \cdot \\
& & & & -C_{i, N}
\end{array}\right]\left[\begin{array}{c}
y_{i, 1} \\
y_{i, 2} \\
\cdot \\
\cdot \\
y_{i, N}
\end{array}\right] \\
& +\left[\begin{array}{ccccc}
E_{i, 1} & 0 & 0 & \cdot & \cdot \\
0 & E_{i, 2} & 0 & & \\
& 0 & E_{i, 3} & \cdot & \cdot \\
& & & \cdot & \cdot \\
& & & & E_{i, N}
\end{array}\right]\left[\begin{array}{c}
y_{i+1,1} \\
y_{i+1,2} \\
\cdot \\
\cdot \\
y_{i+1, N}
\end{array}\right] . \\
& =\left[\begin{array}{c}
R_{i, 1}-N_{i, 1} y_{i, 0} \\
R_{i, 2} \\
\cdot \\
\cdot \\
R_{i, N}-S_{i, N} y_{i, N+1}
\end{array}\right]=R^{(i)}, \text { say. }
\end{aligned}
$$

\section{Direct Solution of The Difference EquATions}

The matrix-vector equation (equation (5)) can be written as

$$
\mathrm{W}^{(\mathrm{i})} \underline{Y}^{(i-1)}+A^{(\mathrm{i})} \underline{Y}^{(i)}+E^{(\mathrm{i})} \underline{Y}^{(i+1)}=\underline{R}^{(i)}
$$

With diagonal matrices $\mathrm{W}^{(\mathrm{i})}$ and $E^{(\mathrm{i})}$ and tridiagonal matrix $A^{(\mathrm{i})}$ all of order $(N X N)$, and column vectors $\underline{Y}^{(i)}$ and $\underline{R}^{(i)}$ of order $N$. To solve the vector recurrence relation a speculation is made that the $\underline{Y}^{(i-1)}$ vector can be related linearly to the $\underline{Y}^{(i)}$ vector as follows:

$$
\underline{Y}^{(i-1)}=B^{(\mathrm{i})} \underline{Y}^{(i)}+\underline{K}^{(i)}
$$

where the $B^{(i)}$ and the $\underline{K}^{(i)}$ are at present unknown matrices and column vectors respectively. Substituting (7) into (6) gives:

$$
\left(W^{(i)} B^{(i)}+A^{(i)}\right) \underline{Y}^{(i)}=\underline{R}^{(i)}-W^{(i)} \underline{K}^{(i)}-E^{(i)} \underline{Y}^{(i+1)}
$$

so that

$$
\begin{aligned}
& \underline{Y}^{(i)}=-\left(W^{(i)} B^{(i)}+A^{(i)}\right)^{-1} E^{(i)} \underline{Y}^{(i+1)} \\
& +\left(W^{(i)} B^{(i)}+A^{(i)}\right)^{-1}\left(\underline{R}^{(i)}-W^{(i)} \underline{K}^{(i)}\right)
\end{aligned}
$$


But $\left.\underline{Y}^{(i)}=B^{(i+1)} \underline{Y}^{(i+1)}+\underline{K}^{(i+1)}\right)$ thus equating coefficients implies

$$
B^{(i+1)}=-\left(W^{(i)} B^{(i)}+A^{(i)}\right)^{-1} E^{(i)}
$$

and

$$
\underline{K}^{(i+1)}=\left(W^{(i)} B^{(i)}+A^{(i)}\right)^{-1}\left(\underline{R}^{(i)}-W^{(i)} \underline{K}^{(i)}\right)
$$

For $i=0$ this gives

$$
\underline{Y}^{(0)}=B^{(1)} \underline{Y}^{(1)}+\underline{K}^{(1)}
$$

To determine the $\underline{K}^{(1)}$, if the first iterate $B^{(1)}=0$ then $\underline{K}^{(1)}=\underline{Y}^{(0)}$.

The matrix and vector sequences are now defined by equations (8) and (9) for $i=1$ to $M$. The $\underline{Y}^{(i)}$ vectors are now calculated starting from right to left (as $\underline{Y}^{(\mathrm{M}+1)}$ is known) using:

$$
\underline{Y}^{(M)}=B^{(M+1)} \underline{Y}^{(M+1)}+\underline{K}^{(M+1)}
$$

The diagonal matrices $W^{(i)}$ and $E^{(i)}$ have elements

$$
W^{(i)}=W_{i, j} \text { and } E^{(i)}=E_{i, j} .
$$

The tridiagonal matrix $A$ has entries

$$
\begin{gathered}
A_{i, j}=-C_{i, j}, j=1 \text { to } N \\
A_{i+1, j}=N_{i, j} \text { and } A_{i, j+1}=S_{i, j} \text {, for } j=1 \text { to } N-1 .
\end{gathered}
$$

\section{THE BOUNDARY CONDITIONS}

A Neumann boundary condition on only one bounding wall will be analysed but similar treatment can be applied so that such a condition applies on other surfaces. In this case the vector of unknown y values is extended to include the $j=0$ row (for the top boundary) and $j=N+1$ for the bottom boundary, (as shown in [6]). The difference scheme is now applied over this extended set i.e. the scheme is centered on the point $j=0$, (and $j=N+1$ for the bottom boundary). Considering for the moment only having a Neumann condition on the top boundary, then centering the scheme on $j=0$ will involve the value of $\mathrm{y}$ at $j=-1$, this term is expressed in terms of the value of $y$ at $j=1$ using the known normal derivative, such that:

$$
\frac{y_{i,-1}-y_{i, 1}}{2 \Delta \varphi} \approx\left(\frac{\partial y}{\partial \varphi}\right)_{i, 0}=\text { known exp ression }
$$

so at the mesh point $(i, 0)(i=1,2, \ldots . M)$ the finite difference scheme gives:

$$
\begin{aligned}
W_{i, 0} y_{i-1,0} & -C_{i, 0} y_{i, 0}+E_{i, 0} y_{i+1,0}=R_{i, 0}-N_{i, 0} y_{i,-1} \\
& +S_{i, 0} y_{i, 1}
\end{aligned}
$$

Applying the boundary condition gives

$$
\begin{aligned}
W_{i, 0} y_{i-1,0} & -C_{i, 0} y_{i, 0}+E_{i, 0} y_{i+1,0}=R_{i, 0}-2 \Delta \psi N_{i, 0}\left(\frac{\partial y}{\partial \psi}\right)_{i, 0} \\
& +\left(S_{i, 0}+N_{i, 0}\right) y_{i, 1}
\end{aligned}
$$

Using

$$
\left(\frac{\partial y}{\partial \psi}\right)_{i, 0}=\sqrt{A_{i, 0}^{2}\left(\frac{1}{q_{i, 0}^{2}}-\frac{1}{B_{i, 0}^{2}}\left(\frac{\partial y}{\partial \varphi}\right)_{i, 0}^{2}\right)}
$$

The normal derivative is now known in terms of the prescribed speed which in this case is along the top boundary. The matrix-vector equations become

$$
\begin{aligned}
& {\left[\begin{array}{ccccc}
W_{i, 0} & 0 & 0 & \cdot & \cdot \\
0 & W_{i, 1} & 0 & & \\
& 0 & W_{i, 2} & 0 & \cdot \\
& & & \cdot & \cdot \\
& & & & W_{i, N}
\end{array}\right]\left[\begin{array}{c}
y_{i-1,0} \\
y_{i-1,1} \\
\cdot \\
\cdot \\
y_{i-1, N}
\end{array}\right]} \\
& +\left[\begin{array}{ccccc}
-C_{i, 0} & \left(S_{i, 0}+N_{i, 0}\right) & 0 & \cdot & \cdot \\
N_{i, 1} & -C_{i, 1} & S_{i, 1} & 0 & \cdot \\
& N_{i, 2} & -C_{i, 2} & \cdot & \cdot \\
& & & \cdot & \cdot \\
& & & & -C_{i, N}
\end{array}\right]\left[\begin{array}{c}
y_{i, 0} \\
y_{i, 1} \\
\cdot \\
\cdot \\
y_{i, N}
\end{array}\right] \\
& +\left[\begin{array}{ccccc}
E_{i, 0} & 0 & 0 & \cdot & \cdot \\
0 & E_{i, 1} & 0 & & \\
& 0 & E_{i, 2} & \cdot & \cdot \\
& & & \cdot & \cdot \\
& & & & E_{i, N}
\end{array}\right]\left[\begin{array}{c}
y_{i+1,0} \\
y_{i+1,1} \\
\cdot \\
\cdot \\
y_{i+1, N}
\end{array}\right] \\
& =\left[\begin{array}{c}
R_{i, 0}-2 \Delta \psi N_{i, 0}\left(\frac{\partial y}{\partial \psi}\right)_{i, 0} \\
R_{i, 1} \\
\cdot \\
\cdot \\
R_{i, N}-S_{i, N} y_{i, N+1}
\end{array}\right] \text {. }
\end{aligned}
$$

Similar analysis can be performed if the bottom boundary is to have a Neumann boundary condition as mentioned previously. The technique can also be applied to the case of having the so-called Robin boundary conditions.

\section{PRESCRIPTION OF THE AXIAL AND SWIRL COMPONENTS OF VELOCITY}

Here numerical solutions to inviscid axisymmetric flow with constant vorticity and a swirl velocity will be derived. The axial velocity component $u_{x}(y)$ at inlet will be chosen to be of the form:

$$
u_{x}(y)=G\left(-y^{2}+A \log _{e}(|y|+B) /(4 \mu)\right.
$$


as described in [7] where $G$ represents the pressure gradient, $\mu$ is the dynamic viscosity, $\mathrm{A}$ and $\mathrm{B}$ are constants not to be confused with the $\mathrm{A}$ and $\mathrm{B}$ occurring in the design plane equations. If the following boundary conditions are applied:

$u_{x}\left(y_{1}\right)=u_{1} ; u_{x}\left(y_{2}\right)=u_{2}$ where $u_{1}$ and $u_{2}$ are chosen so that the maximum value of $u_{x}(y), u_{x \max }(y)$, say is a multiple of $u_{1}$ and $u_{2}$ with $y_{1}$ and $y_{2}$ being the two inlet radii (inner and outer respectively), so that

$$
\frac{d u_{x}}{d y}=\frac{G}{4 \mu}(-2 y+A / y)
$$

thus

$$
\frac{d u_{x}}{d y}=0 \text { at } y= \pm \sqrt{A / 2}
$$

so that

$$
u_{x \max }=\frac{G}{4 \mu}\left(-\frac{A}{2}+\frac{A}{2} \log _{e}\left(\left|\frac{A}{2}\right|\right)+B\right)
$$

choosing

$$
u_{1}=u_{2}=\frac{\mathrm{u}_{\mathrm{xmax}}}{\lambda}
$$

where $\lambda>1$, gives

$$
\begin{aligned}
& u_{x \max }=\frac{G}{4 \mu}\left(-\frac{y_{1}^{2}}{2}+A \log _{e}\left(y_{1}\right)+B\right. \\
& =\lambda \frac{G}{4 \mu}\left(-\frac{y_{2}^{2}}{2}+A \log _{e}\left(y_{2}\right)+B\right.
\end{aligned}
$$

eliminating $\mathrm{B}$ above gives

$$
A=\frac{y_{1}^{2}-y_{2}^{2}}{\log _{e}\left(y_{1} / y_{2}\right)}
$$

knowing A, B follows, from

$$
B=\frac{\frac{A}{2}\left(\log _{e}\left(\frac{A}{2}\right)-1-2 \lambda \log _{e}\left(y_{1}\right)\right)+\lambda y_{1}^{2}}{\lambda-1}, \lambda \neq 1
$$

with swirl velocity given by $u_{\alpha}(y)=k y+l / y$, where the $k$ and $l$ are constants, defining the so-called free and forced vortex whirl respectively.. For the case when body forces exist, for example when the effect of the blades are being considered the force is resolved into a component perpendicular do the flow direction, modeling the guiding action of the blades and into a component parallel to the flow direction, modeling viscous effects, these two cases are discussed in [8] for an incompressible fluid.

\section{The Flow Equations in The Physical Plane $(Y, \alpha, X)$.}

Adopting cylindrical coordinates with $y$ being the radial coordinate, $\alpha$ the circumferential and $x$ the axial coordinate, defining velocity components $u_{y}, u_{\alpha}$ and $u_{x}$ with corresponding vorticity components $\omega_{y}, \omega_{\infty}, \omega_{x}$ in the direction of increasing $y, \alpha$ and $x$ respectively, then the equation of motion for homentropic (and no body forces), inviscid, compressible and axisymmetric flow becomes:

$$
\frac{D \underline{u}}{D t}=-\frac{1}{\rho} \underline{\nabla} \cdot \underline{p}
$$

where $\frac{D}{D t}$ is the material derivative? Equation (10) can be written using well known vector identities as:

$$
\begin{aligned}
& \frac{\partial u_{y}}{\partial t}+u_{x} \frac{\partial u_{y}}{\partial x}+u_{y} \frac{\partial u_{y}}{\partial y}-\frac{u_{\alpha}^{2}}{y}=-\frac{1}{\rho} \frac{\partial p}{\partial y} \\
& \frac{\partial u_{\alpha}}{\partial t}+u_{x} \frac{\partial u_{\alpha}}{\partial x}+u_{y} \frac{\partial u_{\alpha}}{\partial y}-\frac{u_{\alpha} u_{y}}{y}=0 \\
& \frac{\partial u_{x}}{\partial t}+u_{x} \frac{\partial u_{x}}{\partial x}+u_{y} \frac{\partial u_{x}}{\partial y}=-\frac{1}{\rho} \frac{\partial p}{\partial x}
\end{aligned}
$$

Furthermore

$$
\left.\frac{\partial \underline{u}}{\partial t}+\underline{(u} \cdot \underline{\nabla}\right) \underline{u}=-\frac{1}{\rho} \underline{\nabla} \cdot p
$$

can be written (once again using an appropriate vector identity as)

$$
\frac{\partial \underline{u}}{\partial t}+(\underline{\omega} \wedge \underline{u})=-\underline{\nabla}\left(\frac{p}{\rho}+\frac{1}{2} q^{2}\right) \cdot
$$

Thus for steady flow Crocco's form of the equation of motion is obtained, i.e.

$$
\underline{(u} \wedge \underline{\omega})=\underline{\nabla} H
$$

where $H$ is the total head defined by $H=\frac{p}{\rho}+\frac{1}{2} q^{2}$.

Calculating the cross product on the left hand side of equation (12), gives

$$
\begin{aligned}
\frac{\partial H}{\partial y} & =u_{\alpha} \omega_{x}-u_{x} \omega_{\alpha} \\
0 & =u_{x} \omega_{y}-u_{y} \omega_{x} \\
\frac{\partial H}{\partial x} & =u_{y} \omega_{\alpha}-u_{\alpha} \omega_{x}
\end{aligned}
$$

In addition for axisymmetric flow the vorticity vector $\underline{\omega}$ becomes

$$
\begin{aligned}
\underline{\omega}=\underline{\nabla} \wedge \underline{u}= & \left\{-\frac{\partial u_{\alpha}}{\partial x}\right\} \underline{y}+\left\{\frac{\partial u_{y}}{\partial x}-\frac{\partial u_{x}}{\partial y}\right\} \underline{\alpha} \\
& +\left\{\frac{1}{y} \frac{\partial y u_{\alpha}}{\partial y}\right\} \underline{x}
\end{aligned}
$$

The equation of continuity is given by

$$
\underline{\nabla} \cdot(\rho \underline{u})=\frac{\partial\left(\rho y u_{x}\right)}{\partial x}+\frac{\partial\left(\rho y u_{y}\right)}{\partial y}=0
$$


so that

$$
\begin{aligned}
\frac{\partial u_{x}}{\partial x}+\frac{\partial u_{y}}{\partial y} & =-\frac{1}{y \rho}\left(u_{x} \frac{\partial(y \rho)}{\partial x}+u_{y} \frac{\partial(y \rho)}{\partial y}\right) \\
& =-q \frac{\partial \log _{e}(y \rho)}{\partial s}
\end{aligned}
$$

or

$$
\eta=-\frac{q^{2}}{B} \cdot \frac{\partial \log _{e}(y \rho)}{\partial \varphi}
$$

and substituting in equation (2) and integrating gives

$$
y \rho A=f(\psi)
$$

that is $\frac{\partial \psi}{\partial n}=\frac{y \rho q}{f(\psi)}[9]$ has shown that

$$
\frac{\partial \psi}{\partial x}=-\frac{y \rho u_{y}}{f(\psi)} ; \frac{\partial \psi}{\partial y}=\frac{y \rho u_{x}}{f(\psi)}
$$

The arbitrary function $f(\psi)$ represents the freedom in the cross stream distribution of $\psi$ and choosing $f(\psi)$ to be unity everywhere $\psi$ can be identified as the usual Stokes stream function:

$$
\frac{\partial \psi}{\partial x}=-y \rho u_{y} ; \frac{\partial \psi}{\partial y}=y \rho u_{x}
$$

With this choice for A, namely $A=1 /(y \rho)$, equation (2) is simply an identity but in order to discuss equation (3) attention must be restricted to isentropic flow of a perfect gas with constant specific heats. In the absence of body forces the equation of motion can be written in Crocco's form as:

$$
\nabla H=T \nabla S+\underline{u} \wedge \underline{\omega}
$$

where $T$ is the gas temperature, $\mathrm{S}$ is the entropy and $\mathrm{H}$ the stagnation enthalpy. The scalar product of the right hand side of Crocco's equation with $\underline{u}$ vanishes by virtue of the isentropic flow assumption and hence $H$ is constant along streamlines, that is along each stream surface of revolution in axisymmetric flow and therefore both $\mathrm{H}$ and $\mathrm{S}$ are functions of $\psi$ alone. A further quantity dependent only on $\psi$ can be obtained by substituting for $\omega_{y}$ and $\omega_{x}$ in the $\alpha$ component of Crocco's equation, equation (12), gives

$$
0=u_{x} \frac{\partial\left(y u_{\alpha}\right)}{\partial x}+u_{y} \frac{\partial\left(y u_{\alpha}\right)}{\partial y} .
$$

Referring to the meridional plane Fig. 1, it may be deduced that

$$
\begin{aligned}
& u_{x}=q \frac{\partial x}{\partial s} ; u_{y}=q \frac{\partial y}{\partial s} \\
& \Rightarrow \frac{\partial}{\partial s}\left(y u_{\alpha}\right)=0 \\
& \therefore y u_{\alpha}=C(\psi)
\end{aligned}
$$

where $q=\frac{d s}{d t}$. A convenient expression for $\omega_{\alpha}$ for use in equation (3) can be obtained from either the $\mathrm{x}$ component of Crocco's equation as here the y-component:-

$$
u_{x} \omega_{\alpha}=T \frac{\partial S}{\partial y}-\frac{\partial H}{\partial y}+\frac{u_{\alpha}}{y} \frac{\partial C}{\partial y}
$$

using the Stokes' stream function this becomes:

$$
\frac{\omega_{\alpha}}{y \rho}=T \frac{d S}{d \psi}-\frac{d H}{d \psi}+\frac{C}{y^{2}} \frac{d C}{d \psi}
$$

which is the required expression to be used in calculation of $B$ according to equation (3).

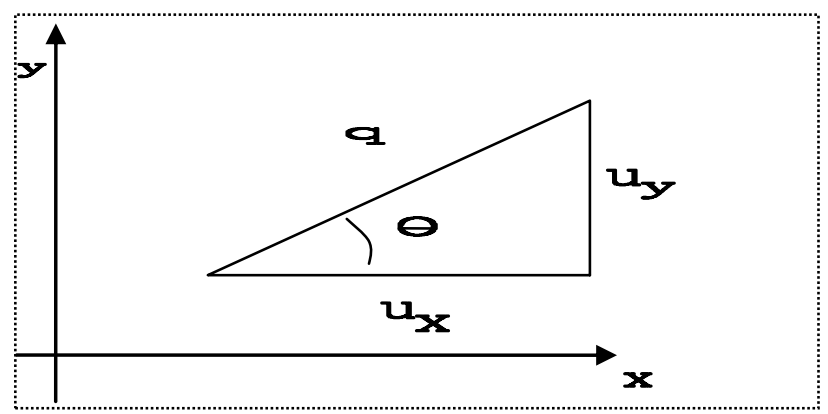

Fig. 1. The meridional plane

\section{Pressure, Density, Speed And Temperature RELATIONS}

Consider a particle of fluid with speed $q_{i}$, pressure $p_{i}$ density $\rho_{i}$ and temperature $T_{i}$ at the point $\left(x_{i}, \alpha, y_{i}\right)$ in the flow field, if $K_{i}$ is a quantity dependent on the entropy $\mathrm{S}$ then at $\left(x_{i}, \alpha, y_{i}\right), p_{i} \rho_{i}^{-\gamma}=K_{i}(S)$, where $\gamma=\frac{c_{P}}{c_{V}}$ with $c_{P}$ and $c_{V}$ being the usual specific heats at constant pressure and volume respectively. Defining the total enthalpy of the gas at $\left(x_{i}, \alpha, y_{i}\right)$ as $H_{i}$, then

$H_{i}=c_{P} T_{i}+\frac{1}{2} q_{i}^{2}$. The ideal gas equation becomes $\underline{p}=R T$ (for one mole) with the additional relations $R=c_{p}-c_{v}$ and, $\gamma=c_{P} / c_{V}$ with the speed of sound $c$ given by $c^{2}=\frac{d p}{d \rho}$. Suppose also that the fluid element is brought adiabatically to rest so that $q_{i}=0$ and by definition of the stagnation values for the pressure, density and speed denoted by the subscript $\mathrm{s}$ then

$$
p_{s} \rho_{s}^{-\gamma}=K_{i}
$$

and

$$
H_{s}=c_{P} T_{s}
$$

as the change is adiabatic it follows that 


$$
H_{i} \equiv c_{P} T_{i}+\frac{1}{2} q_{i}^{2}=H_{s}=c_{P} T_{s}
$$

and

$$
p_{i} \rho_{i}^{-\gamma}=p_{s} \rho_{s}^{-\gamma}
$$

using the Ideal gas equation $\Rightarrow$

$$
T_{i}=\frac{p_{i}}{R \rho_{i}} \text { and } T_{s}=\frac{p_{s}}{R \rho_{s}}
$$

using these last two results in (16) above gives

$$
\frac{c_{P} p_{i}}{R \rho_{i}}+\frac{1}{2} q_{i}^{2}=\frac{c_{P} p_{s}}{R \rho_{s}} \text { and } p_{i}=p_{s}\left(\frac{\rho_{i}}{\rho_{s}}\right)^{\gamma}
$$

so that

$$
\frac{\rho_{i}^{\gamma-1}}{\rho_{s}^{\gamma}}=\frac{1}{\rho_{s}}-\frac{R q_{i}^{2}}{2 c_{p} p_{s}}
$$

leading to

$$
\rho_{i}=\rho_{s}\left[1-\frac{R q_{i}^{2} \rho_{s}}{2 c_{p} p_{s}}\right]^{\frac{1}{\gamma-1}}
$$

so that

$$
\rho_{i}=\rho_{s}\left[1-\frac{(\gamma-1)}{2 \gamma}\left(\frac{\rho_{s}}{p_{s}}\right) q_{i}^{2}\right]^{\frac{1}{\gamma-1}}
$$

using the well known result that $c_{s}^{2}=\not p_{s} / \rho_{s}$ the following results are obtained

$$
\begin{gathered}
\frac{\rho_{i}}{\rho_{s}}=\left[1-\frac{(\gamma-1)}{2} \frac{q_{i}^{2}}{c_{s}^{2}}\right]^{\frac{1}{\gamma-1}} \\
\frac{p_{i}}{p_{s}}=\left[1-\frac{(\gamma-1)}{2} \frac{q_{i}^{2}}{c_{s}^{2}}\right]^{\frac{\gamma}{\gamma-1}} \\
\frac{T_{i}}{T_{s}}=1-\frac{(\gamma-1)}{2} \frac{q_{i}^{2}}{c_{s}^{2}}
\end{gathered}
$$

knowing that

$$
\begin{aligned}
H_{i} & \equiv c_{P} T_{i}+\frac{1}{2} q_{i}^{2}=H_{s}=c_{P} T_{s} \\
& =c_{p} \frac{p_{i}}{\rho_{i} R}+\frac{1}{2} q_{i}^{2}
\end{aligned}
$$

so that

$$
H_{i}=\left[\frac{\gamma}{\gamma-1}\right] \frac{p_{i}}{\rho_{i}}+\frac{1}{2} q_{i}^{2}
$$

which is Bernoulli equation for a compressible fluid ignoring the effects of gravity, [10]. If the flow is isentropic then the quantities $K_{i}$ are the same at all points along a given streamline and the equation set (19) are now relationships along the $i^{\text {th }}$ streamline and not at a specific point $\left(x_{i}, \alpha, y_{i}\right)$, so that

$$
\frac{c_{i, s}^{2}}{\gamma-1}=H(\psi)=\frac{\gamma}{\gamma-1} \frac{p}{\rho}+\frac{1}{2} q^{2}
$$

\section{DOWNSTREAM CONDITIONS}

Downstream a cylindrical flow condition as discussed below will be prescribed. Defining the pressure function $H(\psi)$ and the function $C(\psi)$ as

$$
H(\psi)=\frac{1}{2}\left(u_{x}^{2}+u_{\alpha}^{2}\right)+\frac{\gamma}{\gamma-1} \frac{p}{\rho} \quad \text { and } \quad C(\psi)=y u_{\alpha} \quad \text { for }
$$

cylindrical flow radial equilibrium (from equation (11)) radial component gives

$$
\frac{1}{\rho} \frac{d p}{d y}=\frac{u_{\alpha}^{2}}{y}
$$

Integrating gives:

$$
p-p_{y-\text { inner }}=\int_{y-\text { inner }} \frac{u_{\alpha}^{2}}{y} \rho d y=\int_{y \text {-inner }} \frac{C^{2}(\psi)}{y^{3}} \rho d y
$$

which gives $H(\psi)$ as

$$
H(\psi)=\frac{1}{2}\left(u_{x}^{2}+u_{\alpha}^{2}\right)+\frac{\gamma}{\rho(\gamma-1)}\left(p_{y-\text { inner }}+\int_{\text {yinner }} \frac{C^{2}(\psi) \rho}{y^{3}}\right) d y
$$

which is the required expression in the calculation of the vorticity transported through the duct.

\section{PRESCRIPTION OF THE SPEED Distribution CUBIC IN ARCLENGTH, SALONG THE DUCT}

In this paper the Neumann boundary condition will be prescribed on the top wall boundary so that it is the speed $q$ that is given as a function of arclength along the top boundary. The function chosen to give a the q distribution is chosen to be cubic given by the following piecewise continuous function

$$
\begin{gathered}
q(s)=q_{u}, \text { for } s<s_{1} \\
q(s)=a s^{3}+b s^{2}+c s+d, \text { for } s_{1}<s<s_{2} \\
q(s)=q_{d}, \text { for } s>s_{2}
\end{gathered}
$$

where the constants $a, b, c$ and $d$ are determined such that the cubic $q(s)$ distribution satisfies the following conditions: 


$$
\begin{gathered}
q(s)=q_{u} \text { for } s=s_{1} \\
q(s)=q_{d} \text { for } s=s_{2} \\
\frac{d q}{d s}=\beta(\neq 0), \text { at } s=s_{3},
\end{gathered}
$$

where

$s_{3} \in\left(s_{1}, s_{2}\right)$ and $\beta=\varepsilon\left(\frac{q_{d}-q_{u}}{s_{2}-s_{1}}\right)$, with $\varepsilon \neq 0, s_{1} \neq s_{2}$.

Choosing $s_{3}=\left(s_{1}+s_{2}\right) / 2$ for example gives a symmetrical speed distribution, $\varepsilon$ is an arbitrary scaling multiplier

$$
\frac{d^{2} q}{d s^{2}}=0 \text { at } s=s_{3}
$$
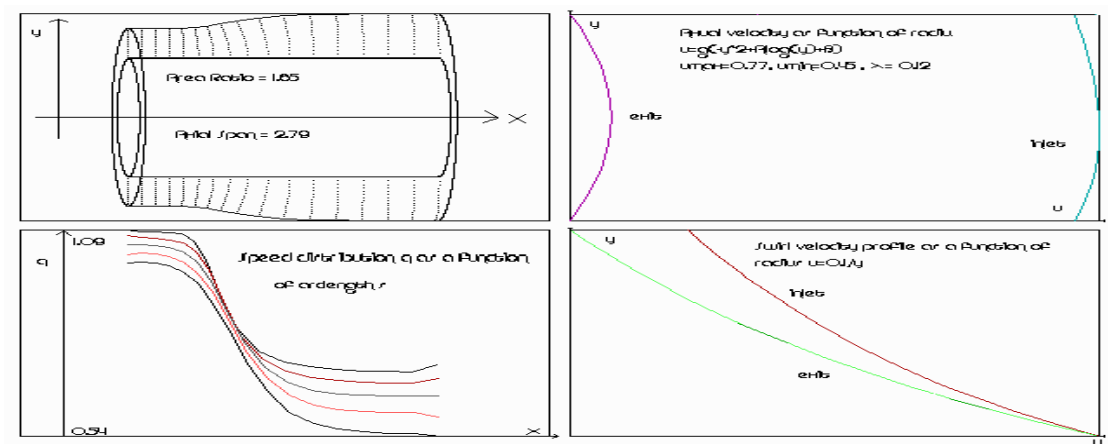

Fig. 2. The geometry and speed distribution (along the top boundary) produced given a swirl velocity given by $u_{x}=G\left(-y^{2}+A \log _{e}(|y|+B) /(4 \mu)\right.$ and

$$
u_{\alpha}=\frac{0.1}{y} u_{\alpha}=0.1 / y \text {. }
$$
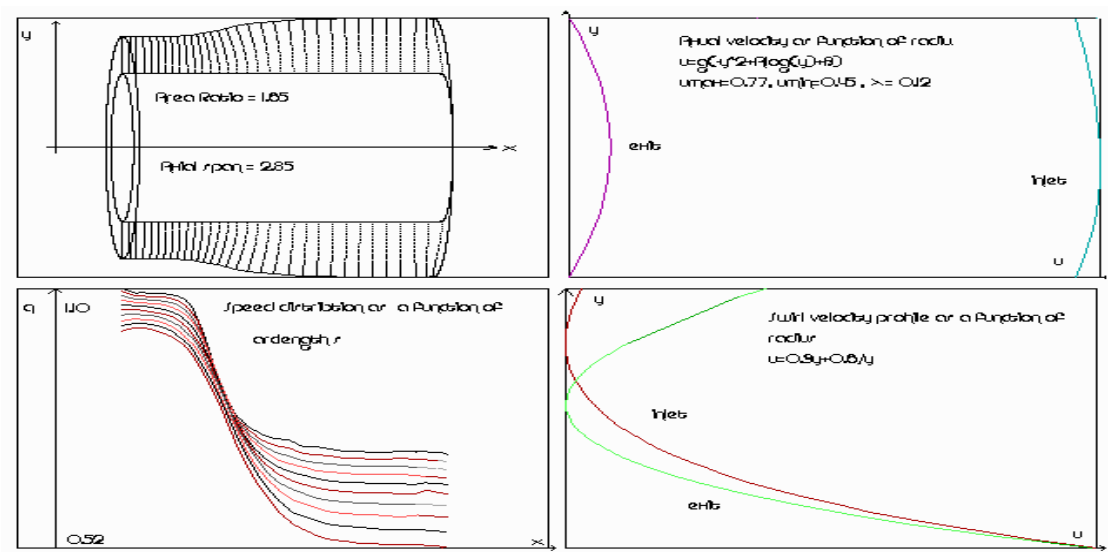

Fig 3. The geometry and speed distribution (along the top boundary) produced given a Swirl velocity $u_{\alpha}=0.3 y+0.6 / y$ and an axial velocity at inlet given by $u_{x}=G\left(-y^{2}+A \log _{e}(|y|+B) /(4 \mu)\right.$.

\section{CONCLUSIONS}

As shown in Fig. 2-Fig. 4 geometries have been produced subject to given upstream and downstream conditions with prescribed Dirichlet boundary conditions. In this case vorticity at inlet has been specified by defining the axial velocity to be of the form

$$
u_{x}(y)=G\left(-y^{2}+A \log _{e}(|y|+B) /(4 \mu)\right.
$$

with swirl velocity given by $u_{\alpha}(y)=k y+l / y$, where the $k$ and $l$ are constants, defining the so-called free and forced vortex whirl respectively. The downstream conditions were such that: cylindrical flow was present. Dirichlet boundary conditions were prescribed however the case with Neumann conditions can be accommodated using the algorithm, in addition so can the case with Robin boundary condition. Further examples of the algorithm with a combination of boundary condition are given in [5], [6], [8], [9]. It was found that at most eight iterations were required to achieve an acceptable level of convergence, with the technique accelerated using Aitken's Method. 

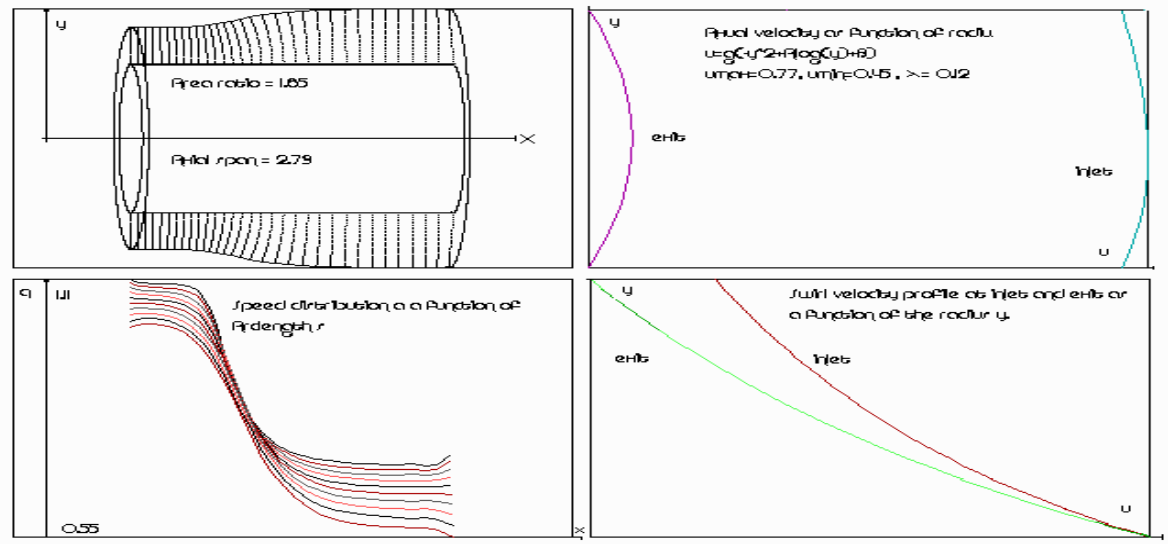

Fig. 4. The geometry and speed distribution (along the top boundary) produced given a Swirl velocity $u_{\alpha}=0.7 / y$ and an axial velocity at inlet given by

$$
u_{x}=G\left(-y^{2}+A \log _{e}(|y|+B) /(4 \mu) .\right.
$$

\section{REFERENCES}

[1] N. Curle and H. J. Davies, Modern Fluid Dynamics, Nostrand Reinhold Company, 1971, ch. I.

[2] J. Cousins, "M-Special Computational problems associated with axisymmetric flow in Turbomachines," Ph.D thesis (CNAA), 1976.

[3] Klier, "M-Aerodynamic Design of Annular Ducts," Ph.D thesis (CNAA), 1990, ch. I.

[4] V. Pavlika, "A Numerical Design Technique associated with the Design of Axisymmetric Ducts for Incompressible Rotational flow," WSEAS International Conferences, Puerto De La Cruz, Tenerife, Canary Islands, Spain, $16^{\text {th }}-18^{\text {th }}$, December 2006, ISSN: 1790-5087, pp. 982-990.

[5] V. Pavlika, "The Calculation of Axisymmetric Ducts geometries for Rotational flow with Blockage effects," Lecture Notes in Engineering and Computer Science, ISBN: 978-988-98671-6, pp. 240-251.

[6] V. Pavlika, "The Design of Axisymmetric Ducts for Incompressible Flow with Blockage Effects and Body Forces," $5^{\text {th }}$ WSEAS International Conference on FLUID MECHANICS (FLUIDS'08), January $25^{\text {th }}-27^{\text {th }}, 2008$, ISBN: 978-960-6766-30-5.

[7] G. K. Batchelor, An Introduction to Fluid Dynamics, Cambridge Mathematical Library, 2000.

[8] V. Pavlika, "The Calculation of Axisymmetric Ducts Geometries for Incompressible flow with Hagen-Poiseuille flow and modeling Body Forces," WorldComp 08, July $14^{\text {th }}-17^{\text {th }}$, 2008, CSREA Press.

[9] V. Pavlika, "The Calculation of Axisymmetric Duct Geometries for Incompressible flow using an Integral Formula based on Green's Theorem," AMAT08 Conference, Memphis, Tennessee, Journal of Concrete and Applicable Mathematics, USA, October $11^{\text {th }}-13^{\text {th }}, 2008$.

[10] M. Thomson, Theoretical Aerodynamics, Macmillan and Co, Limited 1966.

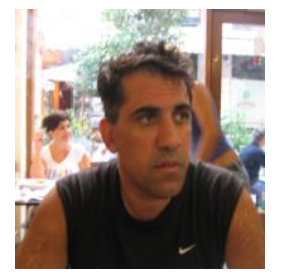

Vasos Pavlika has a B.Sc (Hons) in mathematics and physics (1987), an M.Sc in mathematics (1988), a Ph.D in applied mathematics (1995) and a PGCE in teaching mathematics and physics in Post-Compulsory Education (1999). He has worked at the University of North London (1988), Newham Sixth Form College (1999), Havering College of Further and Higher Education (2000), The University of East London (2000), The London School of Economics and Political Science (2002-present), The University of Gloucestershire (2002), The University of Westminster (2003-2010), SOAS, University of London (2003-Present), The University of Oxford (2004-Present), St George's, University of London (2011-2013). Dr Pavlika is a member of the WSEAS an Editor of the Mathematical Journal of the Antarctica and a member of the WCNA. He has a certificate of Merit for a paper presented at the International MultiConference of Engineers and Computer Scientists 2007, Hong-Kong 21-23 March 2007 and Winner of the Best Paper Award for a paper presented at the International MultiConference of Engineers and Computer Scientists 2007, San Francisco 24-26 October 2007 and has over 20 publications including chapters in the following books: The Calculation of Annular Duct Geometries by Prescribing a Velocity Distribution on the Pressure Surface and the Radius of the Suction Surface”, pp2375-2383, Lecture Notes in Engineering and Computer Science, Newswood Limited 2007, ISBN 978-988-98671-4-0, Magnetostatic field calculations associated with thick Solenoids in the Presence of Iron using a Power Series expansion and the Complete Elliptic Integrals" Applied Numerical Methods, Springer Verlag 2007 and The calculation of axisymmetric duct geometries for incompressible rotational flow with blockage effects and body forces". He advances in Computational Algorithms and Data Analysis, Springer Verlag 2008. His research Interests include: teaching in HE, theoretical and Computational Fluid Dynamics and Magnetostatics. He is a Fellow of the Institute of Mathematics and its Applications (FIMA, CMath, CSci and CMathTeach), a Fellow of the Institution of Analysts and Programmers (FIAP), a Member of the Institute of Physics (MInstP, CPhys), a Fellow of the British Computer Society (FBCS, CITP and CEng), a Member of the Institution of Electrical Engineers (MIEE and MIET) and a Member of the British Society for the History of Mathematics. 